\title{
Nanoscale Liquid is Second Liquid
}

\author{
Boris A. Mosienko \\ Siberian Research Institute of Geology, Geophysics \\ and Mineral Resources, Novosibirsk, \\ Russia
}

\section{Introduction}

In the beginning of the $20^{\text {th }}$ century liquid was considered nonstructural (i.e. similar to a very dense gas). But as it is proved by experiment in 1933, liquid has complicated intermolecular structure [1]. This was the first important broadening of our notions of liquid. From that time on, the liquid structure is studying in many scientific laboratories of the world [2-5]. The second broadening had been developing for a long time in some stages; it is concerned with phase transitions of first kind, in particular, with melting. It turned out that the melting of crystal on its surface begins at the temperature essentially more low than it was considered before. This phenomenon for the first time was noticed and studied by $\mathrm{M}$. Faraday (1850), but the results of his investigations did not gain recognition in that time. The existence of this phenomenon was definitely proved experimentally in 1985 only [6]; it was named premelting. Premelting of ice enables to interpret plenty of natural phenomena (the flow of glaciers, ice slippery, heaving of frozen ground and so on). Investigations on these subjects are carried out now on a large scale in many countries [7-10].

Author of this article has made an attempt to extend further our notions of liquid [11, 12]. It is considered now that sublimation is a direct transition from solid (crystalline) state of matter into gas. The author has propounded and substantiated the principle of least time for first-order phase transitions [11,13]; it is shown by means of this principle that sublimation goes in two steps through a certain intermediate state in the form of surface film. It is concluded that this film consists of nonstructural liquidlike substance which is a certain antipode of liquid; this liquidlike state of matter is named second liquid [12].

In this work, the mentioned subject is continued and developed. From theoretical reasons, it is assumed that second liquid can exist not only in the lower part of phase plane (on the sublimation curve) but also in its upper part, in all existence area of ordinary liquid. The point comes to the sizes of liquid objects: if only one dimension of a liquid object does not exceed a certain critical size $h_{c}$, this object has to consist of second (not ordinary) liquid. This conclusion ought to be of an important applied significance.

It seems that, logically, notion of second liquid is simple and clear [12]; however, it is uncustomary, and therefore difficult for comprehension. For this reason, and also for a coherence of exposition, we shall dwell upon the second liquid notion in the concise form (section 2). In more detail it is considered in [12].

The new data are set forth in sections 3-5. 


\section{Preliminary information}

\subsection{Second liquid}

It is believed at present that the phase transitions $G \rightarrow L, L \rightarrow S$, and $G \rightarrow S$ happen owing to forces of intermolecular interactions, which are called Van der Waals forces. These forces are due to the quantum mechanism (proposed by F. London in 1929).

As known, intermolecular potentials have only one minimum. Since Van der Waals forces are assumed to be the only mechanism of intermolecular interaction, it is reasonable to expect that gaseous molecules should always condense immediately into the solid state (which happens, according to the existing notions, if the process goes along the line 2-2 in Fig. 1). A question arises: Why does liquid exist?

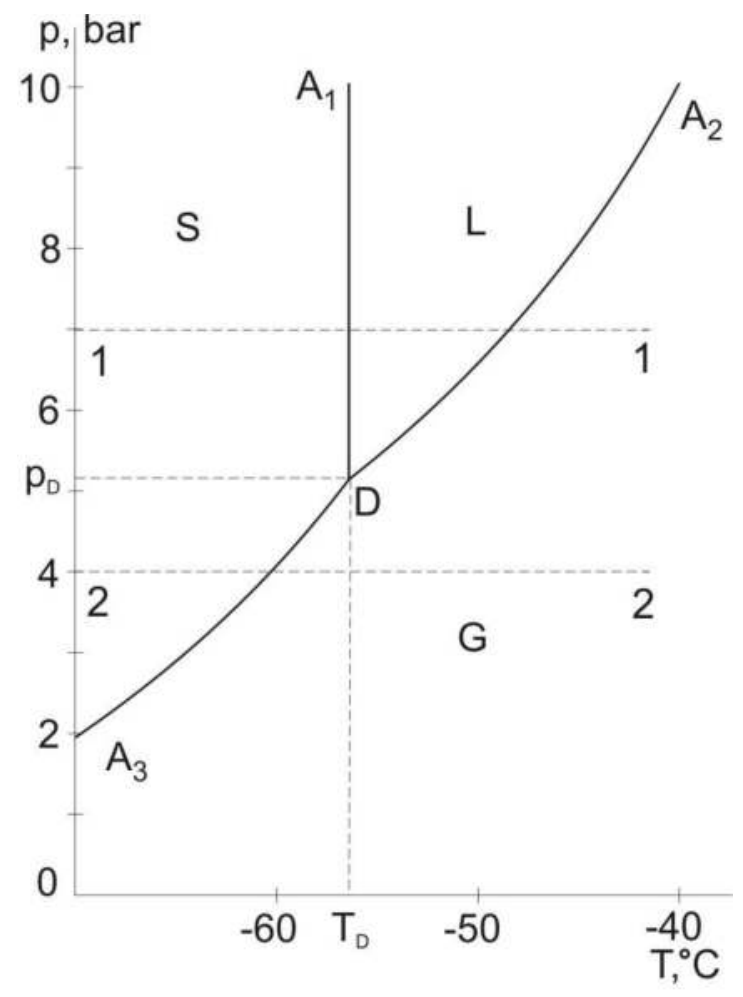

Fig. 1. Typical phase diagram (of carbon dioxide) in coordinates $\mathrm{T}$ (temperature) and $\mathrm{p}$ (pressure). $\mathrm{D}$ is the triple point; $\mathrm{DA}_{1}, \mathrm{DA}_{2}$ and $\mathrm{DA}_{3}$ are the curves of melting, boiling and sublimation, respectively; S, L and G are the areas of existence of solid (crystal), liquid and gaseous states of matter. 
This question is not as naive as it seems at first sight. Indeed, based on the current notions, molecules of gas, in the upper part of the diagram (if the process goes along the line 1-1), descend to the same potential well in two stages: during the $G \rightarrow L$ and $L \rightarrow S$ transitions. Of course, it is hardly the case. Therefore, we assume that, probably, two different independent mechanisms of intermolecular interaction exist, which are realized by the phase transitions $G \rightarrow L$ and $L \rightarrow S$. Let us denote them by symbols $\mathrm{M}_{1}$ and $\mathrm{M}_{2}$, respectively.

Since the mechanisms of intermolecular interaction are independent, they apparently have different physical nature and cannot be combined into a single mechanism at the triple point of phase diagram. So the curves of melting and vaporization do not merge but intersect at the triple point.

It is quite easy to understand that the mechanisms of phase transition $\{G \rightarrow S\}$, in the upper and lower parts of the phase plane, are realized in different successions. Depending on which mechanism is the first to "switch on" by the transition $G \rightarrow S$, the intermediate state can be either an ordinary liquid $\mathrm{L}_{1}=\mathrm{L}$ (as it happens in the upper part of the phase plane) or another modification of liquid which may naturally be called a second liquid $\mathrm{L}_{2}$. As the states $L_{1}$ and $L_{2}$ are generated by different physical mechanisms, the second liquid must substantially differ from ordinary liquid by its characteristics.

\subsubsection{Second liquid of helium}

As known, at a temperature of $4.2 \mathrm{~K}$ under normal pressure, gaseous helium turns into a liquid (helium 1), but at $2.2 \mathrm{~K}$ helium 1 transforms into another modification of liquid which is called helium 2. Helium 1 is an ordinary liquid which differs from other liquids by nothing but low temperature. Helium 2, on the contrary, has unusual properties, the most interesting of which is superfluidity. By its anomalous properties helium 2 radically differs not only from helium 1 but also from all other liquids in nature. However, what is stranger is the very existence of two quite different liquids of helium. The existence of helium 2, the second liquid of helium, is an exception to the rule, which displays itself as a unique physical and logical anomaly.

Given that a second liquid exists, this anomaly is classified as a norm.

The existence of helium 2 is an established fact; meanwhile, the existent idea of sublimation reflects only the level of our present-day knowledge. The facts are admitted to be of greater logical weight than theoretical notions. So, it should be more correct to agree that our notion of sublimation, rather than the existence of second liquid of helium, is a real anomaly.

Now we demonstrate that the phase diagram of helium is a typical diagram of substance with a bulk second liquid.

Formal phase transition curves for different mechanisms of molecular interaction are shown in Fig. 2a. Since these mechanisms are independent, the curves after intersection continue to keep their course and divide the phase plane into four sections. The point $\mathrm{D}$ turns out to be not triple but quadruple. The area of existence of second intermediate state, second liquid $\mathrm{L}_{2}$, together with that of liquid, $\mathrm{L}_{1}$, appears on the phase plane.

Simple thermodynamic reasoning implies that the quadruple point D cannot actually exist; it will fall apart into two triple points $\mathrm{D}_{\mathrm{S}}$ and $\mathrm{D}_{\mathrm{G}}$, connected by a line of the second-order phase transition $\mathrm{L}_{1} \underset{\leftarrow}{\rightleftarrows} \mathrm{L}_{2}$ (Fig. 2b). 

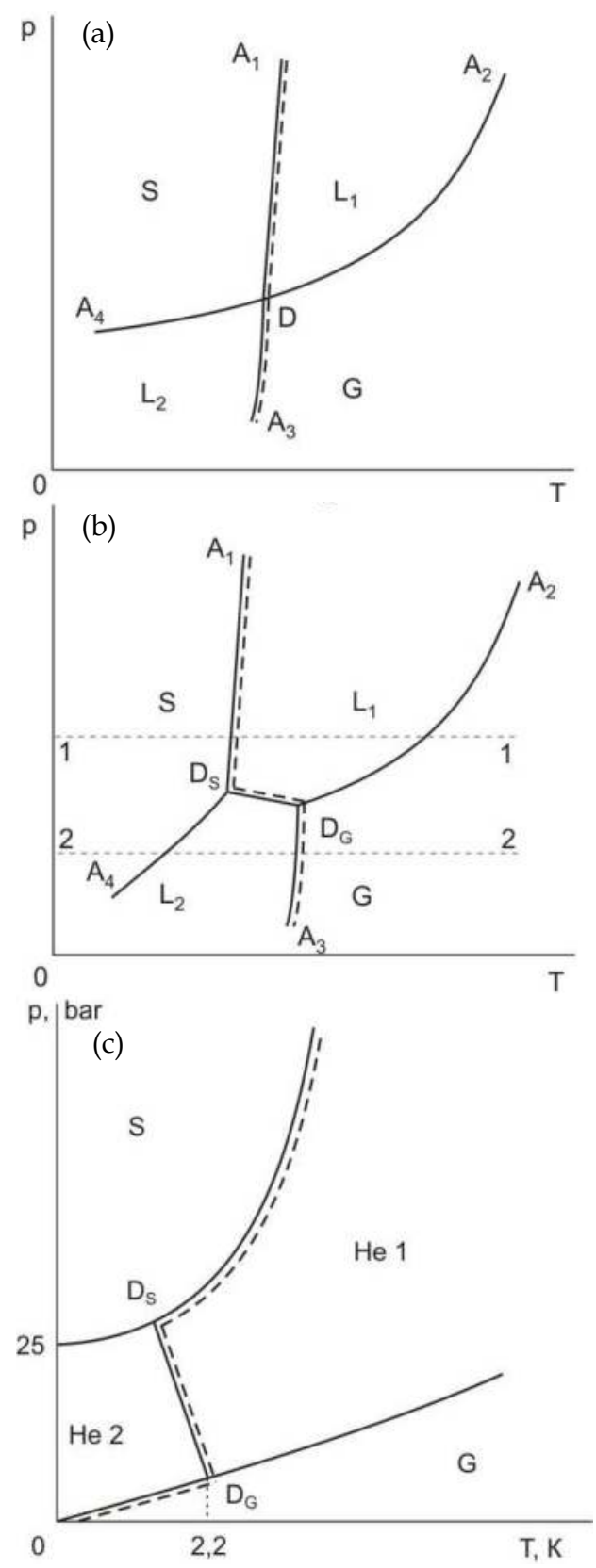

Fig. 2. Scheme explaining the origin of second liquid. a. Diagram with the quadruple point D; $\mathrm{A}_{2} \mathrm{~A}_{4}$ and $\mathrm{A}_{1} \mathrm{~A}_{3}$ are the lines of "switching-on" of the mechanisms $\mathrm{M}_{1}$ and $\mathrm{M}_{2}$, respectively. $\mathbf{b}$. Diagram with a quadruple point fallen apart into the triple points $D_{S}$ and $D_{G}$. c. Helium phase diagram (with vertical exaggeration of scale for clearness). 
It is reasonable to suppose that near the point $\mathrm{D}_{\mathrm{S}}$ potential barriers of the phase transitions $S \rightarrow L_{1}$ and $S \rightarrow L_{2}$ are equal; so, there ought to be no sharp bend of the curve $A_{1} D_{S} A_{4}$ at the point $D_{S}$. The same is true for the point $D_{G}$. Therefore, the phase diagram resembles that of helium (Fig. 2c).

With the area of existence of second liquid tending to zero, we obtain, in the limit, a phase diagram with second liquid in the form of surface film. Obviously, this diagram is similar to that of $\mathrm{CO}_{2}$ (Fig 1).

\subsection{Classical version of superfluidity}

The superfluidity phenomenon was discovered by P. L. Kapitsa in 1938 and in just three years it was interpreted in theory proposed by L. D. Landau. This theory (supported by quantum-mechanical premises) has gained recognition, since it has satisfactorily explained the results of experiments with superfluid helium 2.

Meanwhile, atoms and molecules (as opposed to electrons) are commonly known as quite classical objects. That is why we may expect that the superfluidity phenomenon can be explained in the context of classical physics by the behavior of molecules, namely by the molecular-kinetic theory.

Let us assume that the expression "liquid structure" is clear and well-known. We denote the structure property by symbol Con (from the word "connection") and accept it as characteristic property of liquid.

Next we consider the mechanism of internal friction (viscosity) of flowing liquid or gas. It consists in the following: the neighboring layers which move with different velocities exchange impulses; these impulses are transferred by particles which diffuse through the boundary between layers. Besides, the intermolecular bonds of structural elements (normally - i.e., perpendicularly - orientated to the sliding surface) deform and tear up; this is the second factor of viscosity inherent to liquid only.

It is easy to comprehend (at least, formally) that if the above factors, which condition the mechanism of liquid viscosity, are eliminated, we would get a certain model of superfluidity. Let us show that such a model is possible.

We know that by the transition $\mathrm{L} \rightarrow \mathrm{S}$, which is the result of the mechanism $\mathrm{M}_{2}$, molecules, in a sense, really come to a stop: by crystallization molecules are localized, and diffusion of molecules practically ceases. Of course, it is only the translational motion of molecules which ceases; the thermal movement continues in the form of vibration of molecules relative to strictly defined localized positions which form crystal lattice. Since the transition $G \rightarrow L_{2}$ is realized through the mechanism $\mathrm{M}_{2}$ as well (see Fig. 2), such localization should occur in this case too. The localization of molecules, apparently, may be considered the characteristic property of second liquid; let us denote it by symbol Loc. Thus, liquid has the property \{Con, $-\}$, second liquid has the property $\{-, L o c\}$, and crystal has the property $\{$ Con, Loc $\}$. Hence, the second liquid is a certain antipode of liquid.

Emphasize that the property Loc suggests that diffusion (or, to tell more exactly, selfdiffusion) of molecules in second liquid is practically absent.

As we see, the second liquid lacks the both factors of viscosity. This allows draw the following heuristic prediction: second liquid should be very different from ordinary liquid by its higher fluidity. 
Note that out of caution we speak here of comparatively high fluidity of second liquid, not of superfluidity. This precaution might seem unwarranted if temperature and pressure are expressed in relative form: $T^{*}=T / T_{D}$ and $p^{*}=p / p_{D}$. The combination of inequalities

$$
\mathrm{T}^{*}<1 \& \mathrm{p}^{*}<1
$$

might be considered as the existence condition for second liquid.

The condition (1) is common for all substances. It may be proposed that the property of superfluidity is caused by the low relative temperature $\mathrm{T}^{*}$ (not by the low absolute temperature $\mathrm{T}$, as it is presently assumed). In such definition the assumption of second liquid superfluidity of any substance looks more acceptable.

Note that this point of view could lead to the significant simplification of our understanding of the superfluidity physical nature.

\section{This strange second liquid}

Second liquid properties in broad outline were considered in preceding section. But these properties appear somewhat abstract. In this section we shall dwell upon them in more detail and consider them in the frame of habitual notions (fluidity, diffusion, evaporation, and viscosity).

\subsection{Fluidity}

Liquid has the property Con (structuration), but second liquid has not it. How it manifest itself?

The existence of structural elements (clusters) in liquid causes supplementary resistance during the movement through a tube (canal, capillary); this resistance increases with a diminution of the tube cross-section. In very thin tubes (nanocapillaries), if their diameters are compared with the sizes of structural elements, ordinary liquid apparently could not flow. Meanwhile, the second liquid fluidity, with a diminution of the tube cross-section, even increases owing to the reduction of turbulence.

\subsection{Diffusion and evaporation}

Second liquid has the property Loc (localization), but ordinary liquid has not it. What this lead to?

It is known, that the molecule diffusion of ordinary liquid is a cause of evaporation. As second liquid lacks of diffusion (see section 2.2), one might believe that the rate of its evaporation is much less than that of liquid. By this property, second liquid radically differs from ordinary liquid. Let us accept (as a postulate) the assumption that second liquid does not evaporate at all by temperature $\mathrm{T}<\mathrm{T}_{\mathrm{vap}}$, where $\mathrm{T}_{\mathrm{vap}}$ is the boiling heat.

Superfluidity is the wonderful and extraordinary phenomenon, but it does not arouse a doubt because it is corroborated with the direct experiment carried out at first by P. L. Kapitsa. Lack of evaporation from the second liquid surface is a different matter. It presents itself not only wonderful but impossible phenomenon too. Really, it is difficult to imagine a liquid which does not evaporate from the free surface. An unaccustomed thing perfectly can 
be taken for the impossible one; therefore, a doubt arises: is it like this indeed? That's why we adduce one more argument based on the superfluidity.

It is known, that viscosity of liquid is in a direct dependence upon the diffusion coefficient. If the molecules of helium 2 were capable to diffuse (and, therefore, to evaporate), the viscosity of helium 2 could not be equal to zero; it means that superfluidity could not exist. However, as we know, it does exist.

\subsection{The third condition of superfluidity}

It is obvious, that the presence of the property Loc and the lack of the property Con are necessary for superfluidity. But there is one more obvious and important condition for superfluidity: molecules of second liquid must have the central symmetry; ideally, they must be spherical (as it takes place for helium). For this reason, the most perspective for superfluidity in the bulk phase are elements of the eighth group (neon, argon and so on), whose molecules (atoms) have the central symmetry. Nonspherical molecules (for instance, the lineal molecules $\mathrm{CO}_{2}$ : $\mathrm{O}-\mathrm{C}-\mathrm{O}$ ) cannot bring superfluidity, as they are a cause of viscosity.

\section{Dimension factor}

\subsection{Theoretical considerations}

The notion of liquid as a state of substance with a certain intermolecular structure has almost the century history. This notion has appeared at the definite stage of experimental technology development and continued to get more accurate with its improvements [5]. Emphasize that structural elements of liquid are substantially three-dimensional objects.

Let us carry out an imaginary experiment. Consider the very beginning of melting process (in the ideal case) when on the crystal surface, at first, the film with a thickness $h$ of one molecule appears, then of two molecules, of three molecules and so on. Let us put a question: at which stage (i.e., by which critical thickness $h_{c}$ of a film) do the structural elements of liquid arise? We consider, for the simplicity, that the structure elements are only isometric clusters which can be approximated by spheres of a certain diameter $d_{\mathrm{cl}}$. It is obvious, that the relation $d_{\mathrm{cl}}>d_{\mathrm{mol}}$, where $d_{\text {mol }}$ is the diameter of molecule, take place even for the very small clusters. The clusters cannot arise till the film thickness is smaller of cluster diameter; they simply could not get in. Moreover, one must take into account that the formation of three-dimensional structure elements of liquid is a statistical process; it is possible only when a certain freedom of the movement of molecules in the all three dimensions takes place. Taking into consideration this condition (dimension factor), we come to the following inequality:

$$
h_{c}>d_{\mathrm{cl}}
$$

i.e., the critical thickness must be some times as much as the cluster diameter; let it be by an order of magnitude greater. The diameter of simple molecules is equal $0.5 \mathrm{~nm}$. It means that the diameter of the minimal cluster is equal $1 \mathrm{~nm}$. So, the minimal critical thickness of film is equal $10 \mathrm{~nm}$. Let the maximal one be $50 \mathrm{~nm}$.

Thus, the intermolecular structure of the liquid film can form only when the film thickness reaches a certain critical size $h_{c}$, which might be estimated on the order of some tens of nanometer; let it be $50 \mathrm{~nm}$. The exact value of it could be stated only by experiments. One 
might put a question: what liquid is the film made of, when $h<h_{\mathrm{c}}$ ? As one might see in the case of helium, liquid has only two modifications [12] - $\mathrm{L}_{1}$ (ordinary structural liquid) and $\mathrm{L}_{2}$ (second nonstructural liquid). To introduce any third modification for consideration would be superfluous: this would be a violation of the principle of simplicity. Therefore, the answer is definitive: when $h<h_{c}$, the film consists of second liquid $\mathrm{L}_{2}$.

So, at first, during melting, the film of nonstructural second liquid is formed on the surface of crystal substance. When the film thickness gets equal $h_{c}$, the phase transition of second kind happens, and second liquid transforms into ordinary structural liquid.

It is obvious, that similar transformation happens with drop growing. The growth stages of liquid drops by condensation of vapor are shown in Fig. 3.

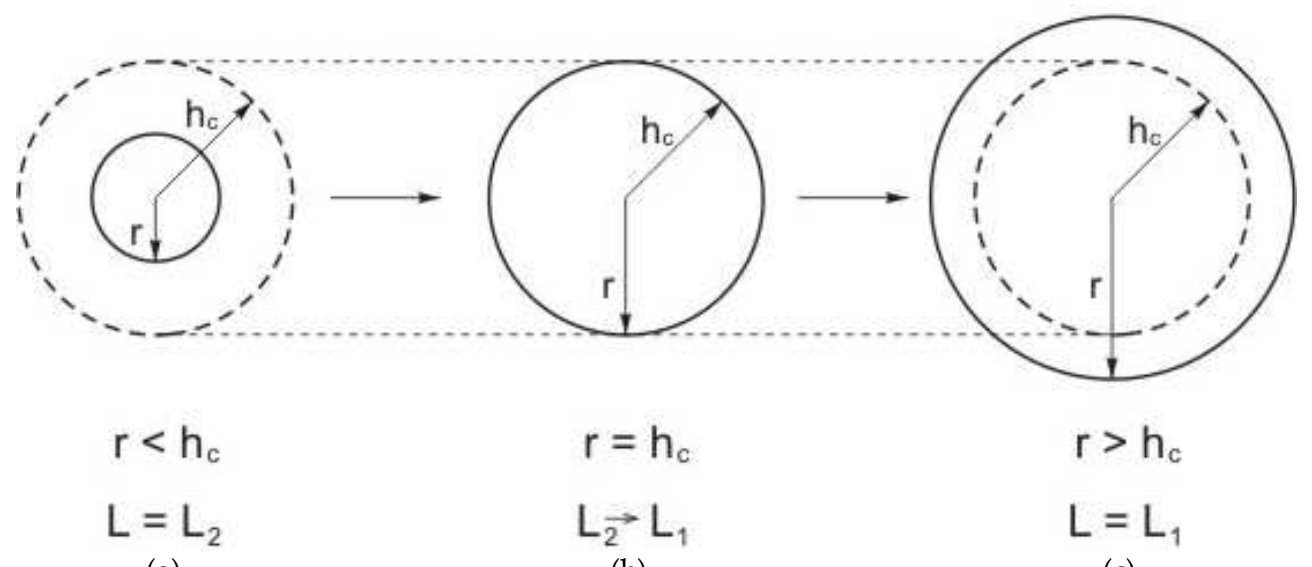

(a)

(b)

(c)

Fig. 3. Growth stages of drops in the upper part of phase diagram. a. A very small drop (nanodrop), $r<h_{c}$; the drop consists of second liquid $\mathrm{L}_{2}$. b. A drop grown up to the critical size, $r=h_{c}$; the phase transition $\mathrm{L}_{2} \rightarrow \mathrm{L}_{1}$ takes place. c. A drop grown out the critical size (microdrop), $r>h_{c}$; the drop consists of liquid $\mathrm{L}_{1}$.

Thus, we have come to a new approach in our conception of liquid. We formulate this conclusion in the general form.

Let us perceive the expression "physical D-space" as an aggregate of $n$ physical bodies ( $n$ is any natural number) which have the same dimensionality. Consider, that physical D-space is incomplete-dimensional, if only one dimension does not exceed $h_{\mathrm{c}}$ (we name such dimensions "truncated"). Below we state the following postulates of the new conception of liquid.

1. There are two modifications of three-dimensional physical D-spaces of liquid: structural liquid $\mathrm{L}_{1}$, in the upper part of the phase plane, and nonstructural second liquid $\mathrm{L}_{2}$ in the lower one (see Fig. 2c).

2. The incomplete-dimensional physical D-spaces of liquid - two-dimensional (nanofilms), one-dimensional (nanothreads) and zero-dimensional (nanodrops) - exist only in the form of nonstructural second liquid $\mathrm{L}_{2}$ (Fig. 3a). Or else, in the concise form: any nanoscale liquid is second liquid. Note that this formulation might be considered a definition of the notion "dimension factor". 
3. When the truncated dimensions of objects of the incomplete-dimensional physical Dspace of liquid, in the upper part of phase plane, increase and reach the critical size $h_{c}$, the second-order phase transition $\mathrm{L}_{2} \rightarrow \mathrm{L}_{1}$ happens, i.e. second liquid transforms into ordinary liquid (Fig. 3b).

\subsection{On the size correlation of solid and liquid nanoobjects}

As known, solid nanomaterials have unusual, unique physical and chemical properties. They contain structural elements, whose geometrical sizes, if only in one dimension, do not exceed the value $h_{\mathrm{o}}=100 \mathrm{~nm}$; it is, first of all, nanoparticles (zero-dimentional elements) and nanofilms (two-dimensional elements).

The above-mentioned critical size of liquids $\left(h_{\mathrm{c}}=50 \mathrm{~nm}\right)$ lies in the accepted nanometric range $(1-100 \mathrm{~nm})$. Note that the cited here sizes ( $50 \mathrm{~nm}$ and $100 \mathrm{~nm}$ ) are tentative; the value $h_{\mathrm{c}}$, by somewhat different estimation, perfectly well could be equal $100 \mathrm{~nm}$ and even more.

Imagine that we have the concrete and sufficiently exact sizes $h_{\mathrm{c}}$ and $h_{0}$ of a certain chemical substance, obtained experimentally. In which relation could be these sizes? Let us assume that they must be equal. This assumption has important heuristic contents which might be used to understand and substantiate the uniquity of properties of nanomaterials. Indeed, if one would take this assumption, the accepted nanometric range would acquire a profound physical meaning: it is the existence area of corresponding second liquid.

\section{Second water on the Earth}

Consider, in which phenomena do nanoobjects of water take part. Let us begin with nanofilms.

\subsection{Water nanofilms on the surface of ice}

It has been mentioned above, that melting of crystalline substance begins at temperature which is essentially below the melting temperature $T_{\text {mel }}$. This phenomenon (premelting) is studied already for some decades. It is established with experiments, that premelting begins at the temperature about $0,9 T_{\mathrm{mel}}$, and the liquid film thickness on a crystalline surface increases together with the temperature [6]. According to the new approach, the liquidlike film of premelting consists of second liquid. At $T=T_{\text {mel }}$ the thickness of the film reaches the value $h_{c}$, and the phase transition $\mathrm{L}_{2} \rightarrow \mathrm{L}_{1}$ happens.

Let us appeal to premelting of water. In this case, we certainly recollect the notorious problem "why is ice slippery". We shall not go into the history of it. The last point in this history was put about 15 years ago [14]: ice is slippery because its surface, from the outset, is covered with a thin film of water (premelting). The ice slippery is getting worse perceptibly with a decreasing of the temperature, when the film thickness approaches to zero. At $T<-$ $30^{\circ} \mathrm{C}$ a certain role for the slippery plays also the friction [14].

We make only one essential alteration in this interpretation: the film on the surface of ice (in the course of premelting) consists of second liquid $\mathrm{L}_{2}$, not of ordinary liquid $\mathrm{L}_{1}$. In the light of this alteration becomes clearly, why the ice slippery considerably diminishes in the days of thaw; this phenomenon is well known to the skaters. Indeed, in this case, the phase transition $\mathrm{L}_{2} \rightarrow \mathrm{L}_{1}$ happens in the surface film, and fluidity of the liquid film falls. 
The film of second liquid covers also the surface of snow crystals. So, the slippery of a snowy sleigh-road and ski-track is explained similarly.

\subsection{Water nanodrops in atmosphere}

Note that in any historical period (for example, 50 or 100 years ago), for the people which are far from science, the situation looks as if all mysteries of atmosphere are studied and explained. In reality, it is far not so.

Let us listen to opinion of the prominent physicist R. Feynman. This is what he said on the mechanisms of electric charging in a thunder-cloud: "Nobody exactly knows how they work. We know only that a lightning happens from the thunderstorm (and know, of course, that a thunder happens from the lightning...)". These words were said a half of century ago [15], but they are of present interest. Moreover, they might be attributed to other mechanisms of the thunderstorm. At present, the thunderous electricity phenomenon contains more secrets than positive solutions.

The problem of thunderstorm can be subdivided into three principal items. 1). How arise and accumulate charged particles (ions)? 2). How happens the spatial separation of the electric charges in a cloud? 3). What is the discharge mechanism of the thunder-cloud? We shall deal with the first item only.

There is only one reliable source of the electric charges in troposphere. It is the cosmic radiation; the radio-activity of mining rocks, which play an important part in the lower troposphere stratum, might be also ascribed to this category. The ions accumulate to some balance concentration when the rate of the ion arising in the volume unit (which can be measured by experiments) becomes equal to the rate of their recombination.

But it turned out that this concentration is insufficient to initiate a thunderous discharge the lightning. A series of auxiliary ionization mechanisms was proposed [6, 16-20], but they were hypothetical and therefore could not lead to the satisfactory solution of the problem.

Let us proceed to nanodrops. One may put a question: what we see as looking on a cloud or a dense cover of a fog? Raindrops - that's quite another thing; they are macroscopic objects. As to a cloud (or a fog), it contains microscopic drops of $0.2-100 \mathrm{mc}$ in sizes. One cold see them, each separately, with microscope only. But even with the best optical microscope it is impossible to see drops whose sizes are smaller $0.2 \mathrm{mc}$. As known, we see owing to the reflected light. The very short waves of the visible light have the length about $0.4 \mathrm{mc}$. If the size of a drop is under $0.2 \mathrm{mc}$, the light does not reflect from it; it means that nanodrops are invisible for us.

But this trouble is not the only one. According to the existent theory, nanodrops cannot arise and exist in atmosphere in general.

Indeed, the pressure of saturated vapor over a spherical drop of radius $r$ is equal

$$
p=p_{0}+2 a / r
$$

where $p_{0}$ is the pressure of saturated vapor over the plane, $\alpha$ is the coefficient of a surface tension (Laplas formula). Hence, additional pressure $2 a / r$ over little drops is more than that over large ones. It means that the vapor saturated over large drop can be unsaturated 
over little one. Therefore, very little drops (nanodrops), originated by chance, are unstable; they must evaporate immediately. That's why it is considered, that clouds consist only of microdrops condensed on the comparatively large solid particles (aerosols) which are numerous in troposphere. These particles, from the outset, ensure considerable radius of the curvature for drops developing on their surfaces.

However, the said above applies to the neutral nanodrops only. As to the electrical charged ones, experiments show, that in this case we have quite a different situation.

Consider the results of experiments carried out 50 years ago with mass-spectrometers installed into geophysical rockets. These experiments were fulfilled in mesosphere, at the altitudes $50-90 \mathrm{~km}$. It was found unexpected ubiquity of hydrated ions $\mathrm{H}^{+}\left(\mathrm{H}_{2} \mathrm{O}\right)_{n}$ and $\mathrm{OH}^{-}$ $\left(\mathrm{H}_{2} \mathrm{O}\right)_{n}$ where $n=1,2,3, \ldots[21,22]$. Besides, in the process of investigation, the more and more heavy hydrated ions were discovered. Otherwise, it was observed an initial stage of the growth of water nanodrops. Note that the centers of nucleation are hydrogen and hydroxyl ions. As ions are present in troposphere, a similar process of the origin of nanodrops must happen in this case too.

It is important to note the following. The mentioned observations in mesosphere had shown that at night the concentration of little ions reduced drastically; and it was naturally, since the most ions in mesosphere arise by photons of the harsh (short-wave) ultra-violet radiation coming from the sun. But the concentration of heavy hydrated ions at night remained like that by day. It means, that the charged nanodrops have a high stability in respect to vaporization and recombination. This was unexpected and incomprehensible.

Subsequently, similar phenomenon was discovered in troposphere [23-25]. The distinction was the following: in troposphere, the most centers of nucleation were represented by the charged fragments of molecules of industrial contamination (nitric oxides, ammonia, sulphuric acid and so on). It was obvious that ions play an important role in the production of new aerosol particles (first of all, water nano- and microdrops) in atmosphere. But the problem of stability of the charged water nanodrops is not solved up to now.

Some ideas for solution of this problem were proposed [26-28], but they did not get the proper experimental corroboration. We give the quotation to elucidate the situation which is formed.

"The role of ions in the production of aerosols is among the least understood, but potentially is an important, process in the Earth's atmosphere. Atmospheric and experimental observations have shown that the nucleation of aerosol particles can occur under conditions that cannot be explained by classical nucleation theory" [29].

Let us show, that the new approach to liquid enables to understand and explain the ioninduced nucleation. The point is that nanodrops consist not of ordinary water but of second one. It is shown in section 3 that second liquid practically does not evaporate. Precisely this strange property of second liquid is the key to the problem in question. So, we come to the following.

1. As second liquid does not evaporate, liquid nanodrops are stable to vaporization. Naturally, this takes place for temperatures $\mathrm{T}<\mathrm{T}_{\mathrm{vap}}$ only.

2. An electric charge, which is a centre of nucleation, cannot come out on the surface of water nanodrop because of the lack of diffusion. This means that it cannot recombine 
with a little ion of the opposite sign: it is isolated by the coat of some layers (ligands) of molecules $\mathrm{H}_{2} \mathrm{O}$. Therefore, the charged nanodrops are stable to recombination. Of course, the recombination, after all, happens (at least, after the transition $L_{2} \rightarrow L_{1}$, i.e., when the critical size $h_{c}$ is overcome).

So, "ions are active in continuously generating a reservoir of small thermodynamically stable clusters that can then rapidly grow in the presence of considerable vapors. These results demonstrate that ions probably play an important role in the production of new aerosol particles in the Earth's atmosphere" [29].

The aforesaid does not mean that the problem of arising and accumulation of electric charges in the thunder-cloud is completely solved. However, it is clear, that nanodrops of second water enable to advance in the proper direction.

\subsection{Nanocapillarity}

In this section, other fields connected with second liquid will be considered in the concise form.

As known, the thin tubes (from one to some tens of microns in diameter), along which liquid moves, are called capillaries. For example, most thin blood-vessels of animals or human beings are capillaries. In more broad meaning, capillaries are the thin cavities with the various shapes of cross-sections (for example, the soil pores). We shall consider capillarity just in such broad meaning.

The capillary liquids in our earthly conditions are, first of all, water and water solutions of diverse chemical compositions. Another instance of natural capillary liquid is oil which fills the pores of sedimentary rocks at different depths from the earth surface.

The value $1 \mathrm{mc}$ is usually taken as the lower limit of capillary sizes. It is no mere chance; this value is near to the lower size of bodies which can be seen in optical microscope. So, capillarity in the existent understanding is microcapillarity. But we are interested in nanocapillarity.

Nanocapillary water causes a serious problem by the production of dry (i.e., completely deprived of water) thin powder which is used in technology processes. Usual drying is not effective in this case: some amount of water (in the form of the smallest capillaries between solid particles), by unknown cause, can not be removed. This remaining moisture can be driven off just by roasting.

According to the new approach, this remaining moisture in nanocapillaries (when the width of cavity $h<h_{c}$ ) consists of second liquid. As was mentioned, second liquid is practically deprived of diffusion, i.e., it practically does not evaporate by $\mathrm{T}<\mathrm{T}_{\text {vap. }}$. It can be removed only by $\mathrm{T}>\mathrm{T}_{\text {vap }}$.

(Note that expression "width of cavity" hardly yields to the strict definition. Nevertheless, let us try to give it. The width $h$ of cavity (capillary) in the given place is the maximal diameter $d_{\max }$ of spherical volume which can be inscribe to the cavity (i.e., $h=d_{\max }$ ). Mathematician most likely would consider such definition incorrect; but for physicists and chemists, I hope, it will be quite acceptable.) 


\subsubsection{Second liquid in biology}

Here we confine ourselves to the very general considerations only.

As is known, intercellular and intracellular cavities of living organisms (including organisms of human beings) have nanometrical sizes [30]. The continuous and intensive movement of water solutions takes place in these cavities. In the last decades, researchers have come to a conclusion that water in intracellular space of living organisms has the special, quite unusual and enigmatic properties. This water was called structurated, quasicrystalline and even living water (see, for example, the works by K. Trincher and also B. V. Deriagin).

According to the new approach, the nanocapillary liquid of living organisms is nonstructural second liquid. As second liquid essentially differs from ordinary liquid (in particular, by high fluidity), at least a part of the existing puzzles of inter- and intracellular water has to get natural explanation. Emphasize that we do not exclude also the influence of other factors.

Note, that we are calling second liquid "nonstructural" only in respect to ordinary liquid which has the property Con. In reality, each liquid has a definite structure (Con or Loc); each liquid, in its way, displays a quality which inherent in solid (crystal). Otherwise, the both liquids are quasicrystalline, but prefix "quasi" should be understood differently, depending on that which liquid is meant.

\section{Conclusion}

Thus, the liquid objects, which have if only one dimension of nanoscale size, consist of second liquid. This conclusion is obtained from theoretical considerations and is of great applied significance. It is shown that the second liquid conception could throw light upon the following problems: 1) the cause of uniquity of solid nanomaterial properties; 2) the ioninduced nucleation in atmosphere; 3 ) some unusual properties of liquid water in nanocavities (particularly, in the cells of living organisms). Apparently, this list might be continued.

It is possible, that the reader had perceived that the third modification of incompletedimensional physical D-space of liquid - nanothreads - was not considered here. The point is that nanothreads, unlike nanofilms and nanodrops, can exist only in very nonequilibrium conditions. Therefore, nanothreads must be found in nature more rarely than nanodrops. It might be supposed that nanothreads, together with nanodrops, play the determinative role in the phenomenon of thunderstorm. But this is a different theme, it requires a special consideration.

\section{References}

[1] J.D. Bernal and R.H. Fowler, J. Chem, Phys. 1, 515 (1933).

[2] J.I. Frenkel, Kinetic theory of liquid, Oxford: Oxford University Press (1946).

[3] D. Turnbull, J. Chem. Phys. 20, 411 (1952).

[4] J.A. Barker, Lattice theories of liquid state, Oxford: Pergamon Press (1963).

[5] H. Reichert, O. Klein, H. Dosh, M. Denk, V. Honkimäki, T. Lippmann, and G. Reiter, Nature (London) 408, 839 (2000).

[6] J.G. Dash, H. Fu, and J.S. Wettlaufer, Rep. Prog. Phys. 58, 115 (1995) and references therein. 
[7] S. Engemann, H. Reichert, H. Dosh, J. Bilgram, V. Honkimäki, and A. Snigirev, Phys. Rev. Lett. 92, 205701 (2004).

[8] N. Materer, U. Starke, A. Barbieri, M.A. Van Hove, G.A. Somorjai, G.J. Kroes, and C. Minot, J. Phys. Chem. 99, 6267 (1995).

[9] X. Wey, P.B. Miranda, C. Zhang, and Y.R. Shen, Phys. Rev. B 66, 085401 (2002).

[10] H. Bluhm, D.F. Ogletree, C.S. Fadley, Z. Hussain, and M.G. Salmeron, J. Phys. Condens. Matter. 14, L227 (2002).

[11] B.A. Mosienko, Z. Phys. Chem. 222, 1533 (2008).

[12] B.A. Mosienko, Z. Phys. Chem. 223, 905 (2009).

[13] B.A. Mosienko, Z. Phys. Chem. 224, 1347 (2010).

[14] R. Rosenberg, Phys. Today 58, issue 12, 50 (2005).

[15] R. Feynman, R. Leiton, and M. Sands, The Feynman lectures on physics, Vol. 2, Chap. 5, London: Addison-Westley Pub. Co. (1964).

[16] C.T.R. Wilson, J. Franklin Inst. 208, 1 (1929).

[17] R.G. Fleagle and J.A. Businger, An introduction to atmospheric physics, New York: Academic (1980).

[18] T. Takahashi, J. Phys. Chem. 87, 4122 (1983).

[19] G.J. Turner and C.D. Stow, Phil. Mag. 49, L25 (1984).

[20] Y. Dong and J. Hallett, J. Geophys. Res. 97, 20361 (1992).

[21] G. Brasseur and S. Solomon, Aeronomy of the middle atmosphere, Dordrecht: D. Reidel Pub. Co. (2005).

[22] A.D. Danilov, Common aeronomy, Leningrad: Hydrometeoizdat (1978).

[23] D. Smith and P. Spanel, Mass Spectr. Rev. 14, 255 (1995).

[24] S.H. Lee, J.M. Reeves, J.C. Wilson, D.E. Hunton, A.A. Viggiano, T.M. Miller, J.O. Ballenthin, and L.R. Lait, Science 301, 1886 (2003).

[25] K. Nagato, C.S. Kim, M. Adachi, and K. Okuyama, J. Aerosol Sci. 36, 1036 (2005).

[26] F. Arnold, Nature 284, 610 (1980).

[27] F. Raes, A. Janssens, and R. Dingenen, J. Aerosol Sci. 17, 466 (1986).

[28] R.P. Turco, J.X. Zhao, and F. Yu, Geophys. Res. Lett. 25, 635 (1998).

[29] H. Svensmark, J.O.P. Pedersen, N.D. Marsh, M.G. Enghoff, and U.I. Uggerhoj, The Royal Society, Online ISSN, 1471 (2006).

[30] A.R. Rees and M.J.E. Sternberg, From cells to atoms, Chap. 1, 3, Oxford, London and so on: Blackwell Scientific Pub. (1984). 


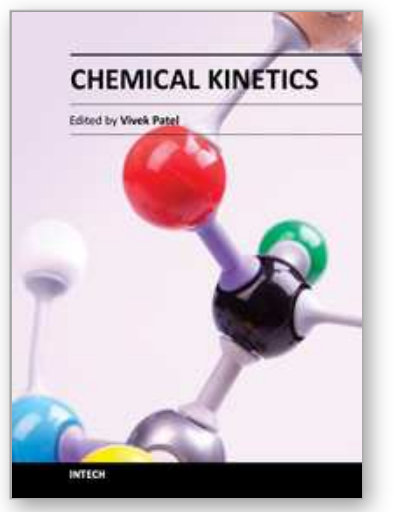

\author{
Chemical Kinetics \\ Edited by Dr Vivek Patel
}

ISBN 978-953-51-0132-1

Hard cover, 344 pages

Publisher InTech

Published online 29, February, 2012

Published in print edition February, 2012

Chemical Kinetics relates to the rates of chemical reactions and factors such as concentration and temperature, which affects the rates of chemical reactions. Such studies are important in providing essential evidence as to the mechanisms of chemical processes. The book is designed to help the reader, particularly students and researchers of physical science, understand the chemical kinetics mechanics and chemical reactions. The selection of topics addressed and the examples, tables and graphs used to illustrate them are governed, to a large extent, by the fact that this book is aimed primarily at physical science (mainly chemistry) technologists. Undoubtedly, this book contains "must read" materials for students, engineers, and researchers working in the chemistry and chemical kinetics area. This book provides valuable insight into the mechanisms and chemical reactions. It is written in concise, self-explanatory and informative manner by a world class scientists in the field.

\title{
How to reference
}

In order to correctly reference this scholarly work, feel free to copy and paste the following:

Boris A. Mosienko (2012). Nanoscale Liquid is Second Liquid, Chemical Kinetics, Dr Vivek Patel (Ed.), ISBN: 978-953-51-0132-1, InTech, Available from: http://www.intechopen.com/books/chemical-kinetics/nanoscaleliquid-is-second-liquid-

\section{INTECH}

open science | open minds

\author{
InTech Europe \\ University Campus STeP Ri \\ Slavka Krautzeka 83/A \\ 51000 Rijeka, Croatia \\ Phone: +385 (51) 770447 \\ Fax: +385 (51) 686166 \\ www.intechopen.com
}

\author{
InTech China \\ Unit 405, Office Block, Hotel Equatorial Shanghai \\ No.65, Yan An Road (West), Shanghai, 200040, China \\ 中国上海市延安西路65号上海国际贵都大饭店办公楼 405 单元 \\ Phone: +86-21-62489820 \\ Fax: $+86-21-62489821$
}


(C) 2012 The Author(s). Licensee IntechOpen. This is an open access article distributed under the terms of the Creative Commons Attribution 3.0 License, which permits unrestricted use, distribution, and reproduction in any medium, provided the original work is properly cited. 